QUELQUE RECTIFICATIONS DE NOMENCLATURE CONCERNANT DES MOLLUSQUES DE LA FAUNE PALEARCTIQUE.

\author{
Par M. P. Pallary. \\ Read 12th November, 1920.
}

JE groupe ici un certain nombre de rectifications concernant des Mollusques terrestres de la région paléarctique. Je mentionne même deux corrections déjà faites parcequ'elles sont éparses dans d'autres recueils et ignorées, pour ainsi dire. La plupart de ces rectifications concernent des espèces du nord de l'Afrique: c'est encore une raison qui m'a fait les réunir dans le même article.

Patula annaI, Paladilhe.

Dans une notice intitulée: "Kritische Fragmente," M. P. Hesse a fait observer, en 1915 (Nachr. der Deutsch Malak. Ges., p.53) que d'après l'opinion du regretté J. Ponsonby qui a examiné le type de l'Helix simplicula, Morelet, cette espèce serait identique à Helix annai, Paladilhe.

P. Hesse ajoute que l'espèce de Morelet est un Helicodonta tandis que Westerlund en a fait un Hyalinia à cause de son péristome tranchant.

L'H. simplicula a été décrit en 1845 par Morelet dans: "Descrip. des Mollusques du Portugal," pp. 56, 57, et figuré pl. vi, fig. 2.

Le nom de Paladilhe date de 1875 in "Coq. terr. et fluv. rapp. du Maroc", par le Dr. Bleicher, Revue et Mag. de Zoologie, pp. 82-3.

Ce vocable d'annai déjà, d'ailleurs, été préemployé par Lewis et Semper (vide Pfeiffer, "Monogr. Helic," t. vii, pp. 324 et 527).

\title{
Xerophila aMANDA.
}

L'ingénieur G. Rolland a figuré dans son " Hydrologie du Sahara algérien," 1894, vol. iii, pl. xxviii, fig. 3, une Xérophile sous le nom d'Helix amanda, Rssmlr., qui est celui d'une espèce littorale des environs d'Alger.

Cette identification étant erronée, l'espèce désertique pourra s'appeler : $X$. choisyi du nom du chef d'une mission saharienne.

Le texte de l'ouvrage en question ne donne absolument aucune indication relative à cette intéressante forme.

Xerophila Finitima.

Dans sa notice sur les " Hélices recueillies dans le midi de l'Espagne et au Maroc" (Rev. et Mag. de Zool.), p. 621, Morelet, en 1854, au lieu de conserver à cette espèce le nom de finitima déjà donné par Férussac (bien que sans description) a cru devoir substituer à ce nom déjà connu celui de calpeana! 1

Morelet est d'autant moins excusable d'avoir repris ce nom de

$1 H$. calpeana $=H$. finitima, Fér., in Museum. Synonymie reproduite par Pfeiffer dans le 4e vol. de la Monog. Helic., 1859, p. 188. 
finitima qu'il le mentionne dès 1845 dans son ouvrage sur les "Mollusques du Portugal"; l'espèce, dit il, se trouve dans la collection de Férussac comme provenant de Gibraltar (p. 24). ${ }^{1}$

Mais ce qui est absolument déconcertant c'est que ce même auteur ait cru devoir reprendre en 1880 ce nom de finitima (in "Faune Maroc", p. 39) pour une espèce absolument différente du cap Cantin !

En résumé il faut restituer le nom de $H$. finitima à l'espèce décrite en 1854 par Morelet comme $H$. calpeana.

\section{XEROPHILA PETRICOLA.}

Ainsi que nous venons de l'indiquer le nom de finitima avait étá déjà employé en 1837 par Férussac et indiqué comme synonyme de $H$. calpeana par Morelet en 1854. C'est donc par suite d'un oubli de la part de ce malacologiste que ce même nom a été de nouveau employé par lui en 1880 pour une espèce d'un autre groupe.

Bourguignat, qui, le premier, a signalé cette confusion dans son "Prodrome de la Malac. de la Tunisie", 1883, p. 90, a proposé le nom de cantinica (de la localité originale : cap Cantin) qui a l'inconvénient d'avoir le même radical que celui de cantiana, Montagu.

Westerlund, 1889, in "Fauna paläarct", ii, p. 318, a publié une correction manuscrite faite par Morelet sous le nom de petricola. On a donc, en définitive, la synonymie suivante :-

1880. H. finitima, Morelet (non Férussac): "Faune Maroc," p. 39.

1883. H. cantinica, Bgt.: "Prod. malac. Tunisie," p. 90.

1889. H. petricola, Morelet in sched (Kobelt) in West., " Fauna paläarct., ii, p. 318.

\section{Helix taniata.}

Westerlund a décrit, en 1888 (Bull. de la Soc. malac. France, pp. 58-9) sous le nom de tæniata une Hélice du groupe Xérophile dont j'ai donné une figuration dans le "Journal de Conchyliologie", 1898, pl. vi, fig. 1.

Mais il existait déjà un Helix (Heterostoma) tceniata, Webb et Berthelot, 1833, "Synopsis," etc., p. 324, espèce des Canaries.

De plus d'autres espèces, appartenant à des groupes divers, portaient aussi ce nom de taniata qui leur avait été donné par Reeve, Montrouzier et Megerle von Mühlfeld.

Nous rebaptiserons l'espèce de Westerlund : Xerophila verminiana.

\section{Helix micRomphalus.}

Lowe a publié, en 1852 (Ann. and Mag. Nat. Hist., ix, p. 118), un Helix micromphalus, de Madère, incorporé actuellement dans la section Irus dont il est également l'auteur.

1 Ce nom de fenitima se trouve dans le "Catal. de la Coll. de Férussac", 1837 , p. 5, avec l'indication. Europe Tan. (sans doute Tanger) et le nombre; 10 exemplaires. 
En 1870 Letourneux ("Excurs. malac. Kabylie": Annales de Malacologie, i, p. 304) a décrit un Helix micromphalus dans les. Cette Hélice est considérée comme un Fruticicola.

Nous dédions l'espèce de la Kabylie au savant et regretté Maupas avec qui nous étions lié par une solide amitié. En son souvenir cette Hélicidée s'appellera Helix maupasi.

\section{HELIX TERVERI.}

Il existe dans la nomenclature plusieurs Helix terveri ou terveriana. Mais trois seulement intéressent la faune paléarctique.

Le plus ancien et le plus connu est le Xerophila terveri de Michaud, 1831 ("Compl. Hist. Moll. France," p. 26, pl. xiv, figs. 20-1).

Le deuxième est le Bulimus terveriana, W.-B., 1833 ("Synopsis Moll. terr. Ins. Canarias" : Ann. Sci. Nat., xxviii, p. ), espèce du groupe Cochlicella.

Il faudra donc substituer à ce nom celui de Cochlicella scalarioides, Reeve ("Conch. Icon.," 1850, No. 590, pl. lxxx), qui est strictement synonyme.

Enfin Rossmässler a figuré dans l' "Iconographie", 1856, figs. 816-19, un Helix terveri qui n'est autre que le $X$. adolfi, Pfeiffer, 1854.

\section{Xerophila MOGADORENSIS.}

Dès 1860, Lowe (Journ. Linn. Soc. (Zool.), v, p. 197) a décrit une variété mogadorensis de l'Helix caperata.

La description que donne ce naturaliste au bas de la page 197 ne laisse aucun doute sur l'identité de cette variété mogadorensis avec l'Helix jaylei décrit en 1875 par Paladilhe. Si le doute pouvait encore subsister, la comparaison qu'il fait de cette variété avec la figure 830 a de Rossamässler la léverait immediatement. Il faut donc reprendre le nom de Lowe et modifier celui de mogadorensis attribué par Bourguignat à un Xeroleuca.

On aura donc :-

$$
\begin{aligned}
& \text { 1860. Xerophila mogadorensis, Lowe. } \\
& \text { 1875. X. jaylei, Paladilhe. } \\
& \text { 1875. X. rusticula, Paladilhe. } \\
& \text { 1884. X. alberti, Kobelt. }
\end{aligned}
$$

Quand à l'espèce que Bourguignat a publiée en 1860 sous le nom d'Helix mogadorensis et qui appartient au groupe Xeroleuca, nous lui appliquons le nom de Soürensis qui est la transcription indigène du nom de Mogador (Soüra).

\section{XEROPHILA DISSIMILIS.}

Tout récemment j'ai publié (Bull. Soc. Hist. nat. Afriq. nord., 1918, p. 141) un $X$. dissimilis du Maroc oriental.

Bien que je susse pertinemment que ce nom de dissimilis avait été appliqué antérieurement à des Hélicéens de groupes considérés anjourdhui comme génériques, j'avais crû pouvoir appliquer ce 
qualificatif à une Xérophile puisque la confusion ne me semblait pas possible.

Mais M. Dautzenberg m'a fait amicalement observer qu'il y avait un inconvénient sérieux à se servir de noms déjà employés pour désigner d'autres Hélicéens car, si par suite de modifications de la Nomenclature une espèce venait à passer d'un genre dans un autre, le même nom serait porté par deux espèces.

Me rendant à cette suggestion j'ai nommé $X$. lecointrei (Journ. de Conchyl., 1919, p. 63), mon $X$. dissimilis.

\section{Xerophila omphalodes.}

Pour la même raison j'ai nommé $X$. dzirana (Journ. de Conchyl., 1919 , p. 64) la Xérophile dont j'avais donné la diagnose (Bull. Soc. Hist. nat.Afriq. du nord., 1918, p. 138) sous le nom de X.omphalodes.

\section{Xerophila agenora.}

Dans mon "Catal. de la faune Malac. de l'Egypte", 1909, p. 35, j'ai nomme $X$. gharibounensis une Xérophile appelée $H$. ptychodia par Von Martens, mais très distincte de l'espèce publiée sous ce nom par Bourguignat.

Or ce $X$.gharibounensis est identique au $X$.agenora, West ("Fauna Paläarct, ii, p. 353) comme j'ai pu m'en assurer en comparant le type de cette dernière qui me fût aimablement communiqué par son possesseur feu John Ponsonby.

Les figures des "Conchol. Mitth.", 1889, pl. xxxi, fig. 11 à 13, sont rigoureusement semblables au type du $X$. agenora.

\section{Cochlicella.}

La plupart des auteurs attribuent cette coupe à Risso alors qu'elle est mentionnée dès 1819 dans le "Prodrome" de Férussac, p. 51. On la retrouve dans les: "Tableaux syst. des anim. moll.," 1821, p. 52 et, en 1837, dans le "Catalogue de la coll. Férussac", p. 9.

Risso a adopté cette section et c'est pourquoi on la lui attribué si généralement.

MM. Fagot et Caziot ("Moll. de Corse", 1903, p. 211) donnent la préférence à Elisma, Leach, 1820, teste Turton 1831.

Mais c'est à tort que ces malacologistes affirment que le genre de Férussac comprend des espèces disparates parmi lesquelles il est impossible de choisir un type.

Pour montrer combien l'assertion de MM. Fagot et Caziot est erronée voici la liste des espèces classées dans son onzième sous genre par Férussac :-
Helix conoidea.
H. trochoides.
$H$. ventrosus $=$ ventricosus, Drpd
H. acuta.
H. barbara.
H. oryza. 

H. clavulus.
H. calcarea.
H. decollata.
H. septenaria.

Or la tradition exigeant que l'on prenne pour type d'une coupe la première espèce citée on peut se convaincre que le genre Cochlicella est bien valide puisque les cinq premières espèces au moins constituent un groupe très homogène.

Il faut donc restituer cette coupe à Férussac et lui donner la priorité sur le genre Elisma.

\section{Archelix jourdaniana.}

Ce nom de jourdaniana a été attribué par Bourguignat à une Hélice de la région de Tlemcen ("Moll. nouv. litig.", fasc. viii, 1867, pp. 75-7, pl. xxxviii, figs. 1 à 4).

Mais, comme je l'ai fait observer en 1914 (Nachr. Deutsch. Malak. Ges., p. 20) il existait déjà un Helix jourdani, Michaud (Journ. de Conchyl., 1862, pl. iii, figs. 12-13) espèce fossile du Miocène.

J'ai donc donné à l'espèce actuelle de l'Oranie le nom d' $A$. agadirensis, dérivé de l'ancien nom arabe de Tlemcen.

TaChea Coquandi.

Dans l' "Iconographie", 1920 (tom. xxiii, p. 241), M. P. Hesse a exhumé le nom de litturata, Pfeiffer, 1851, pour remplacer celui, plus connu, de coquandi, Morelet, 1854.

Cette rectification est d'autant plus admissible qu'il existe un Helix coquandiana fossile décrit en 1842 par Mathéron ("Catal. corps organ. fossiles Bouches du Rhône", pl. xxxiii, figs. 5-6). Terre à gypse près d'Aix. Eocène supérieur.

Par contre, c'est par erreur que M. Hesse identifie l' $H$. dillwyniana de Pfeiffer à l'Eremina duroi de Hidalgo (p. 256). L'espèce de Pfeffier est plus probablement un Chloritis.

\section{Buliminus CALLOMPHALUS.}

En 1876 Bourguignat a publié ("Species novissimae", No. 23) un Bulimus euryomphalus, une des espèces les plus remarquables du nord de l'Afrique.

Mais, en 1891 ("CEuvres scientifiques", p. 67), cet auteur (sous le couvert du Dr. Servain) a indiqué, comme meilleur, le nom de callomphalus, parce qu'il existe un Bul. euryomphalus, Jonas, 1844, espèce du Vénézuela.

J'ajoute que le Bul. callomphalus est le type de la section Omphaloconus, West.

\section{Ferussacia atlasica.}

J'ai décrit sous ce nom une Férussacie du grand Atlas (Bull. Muséum hist. nat., 1915, p. 27).

Or ce même nom se trouve dans le "Prodrome Malac. terr. 
Tunisie ", 1885, de Letourneux et Bourguignat (p. 147). Toutefois ce n'est qu'un nom nû.

Mais il ne peut s'agir de la même espèce, un renvoi, au bas de la page, informant le lecteur que cette Férussacie est une espèce du petit Atlas de Blida.

Je nomme donc à nouveau la forme marocaine: Ferussacia derenica.

Adrar n'Deren est le nom berbère du grand Atlas.

J'ai donné une figuration de mon espèce dans le Journ. de Conchyl., 1920, pl. ii, figs. 12 à 14 .

\section{Semilimax.}

Plusieurs auteurs, entre autres le Dr. P. Fischer, Westerlund, attribuent le genre Semilimax à Stabile 1859 (Rev. et Mag. de Zoologie).

Or ce genre a été institué en 1802 par Férussac, père, et est mentionné dans les "Tableaux systém", du fils, 1821, p. 21, en synonymie de son genre Helicolimax.

Hermannsen en fait également mention en le considérant comme synonyme de Vitrina.

Il faudra donc restituer le genre Semilimax à Férussac père.

Planorbis BOISSYi.

Ce nom a été donné à deux espèces : l'une, du bassin de Paris, par Deshayes ("Coq. foss. env. Paris", 1837, pl. xlv, figs. 20-1).

L'autre, par Potiez et Michaud ("Galerie Moll. Douai, 1838-44, i, p. 208, pl. xxi, figs. 4-6) à une espèce actuelle de l'Egypte.

Mais feu Ancey (Journ. de Conchyl., 1903, No. 3, p. 321) a émis l'avis que le $P l$. subsalinarum, Innés, est la même espèce que le $P l$. Boissyi, Pot. et Mich C'est donc ce dernier nom qu'il conviendra d'adopter pour l'espèce actuelle de l'Egypte. (Voir mon "Cat. de la faune malac. Egypte”, 1909, p. 55).

\section{Pythia.}

Les Proceedings d'Octobre, 1919, pp. 136-9, contiennent une intéressante étude de MM. Kennard et Woodward sur les noms génériques des Ellobiidæ britanniques où ils concluent à l'adoption des genres Phytia, Gray, pour le groupe Myosotis et Leuconia, Gray, pour le groupe bidentata.

Or, dans mon "Catal. des Moll. du litt. méditer. de l'Egypte", 1912 (Addit. et correct., p. 187), j'ai écrit ceci :

"Le genre $P$ ythia, Bolten, a pour type le $P$. scarabeus qui est une coquille exotique tout à fait différente.

"C'est le genre Phytia, Gray, 1821, qui a pour type: Auricula myosotis.

"Mais ce nom de Phytia n'est qu'une faute de copie pour Pythia (voir Hermannsen, ii, p. 383). Toutefois ce nom ayant été préemployé par Bolten en 1798 et Schumacher en 1817 ne peut être adopté pour ce genre. 
“D'autre part, quoique le nom d'Alexia de Leach soit de 1818, ce nom est resté manuscrit et n'a été publié qu'en 1847 par Gray. Or, il existait déjà un genre Alexia, Steph., 1835, pour des Coléoptères.

"Enfin le nom de Kochia, Pallary, 1900, dont le type est Alexia denticulata, Montagu, a été également préemployé en 1891 par Frech.

"Il ne reste donc plus pour ce genre que le nom de Myosotella Monterosato, 1906, institué pour les A. Payreaudeaui, A. myosotis, etc."

J'ajoute que le genre Auricella (Brard), in Jurine 1817, qui pourrait être adopté pour le groupe Pythia est primé par Auricella, Hartmann, $1821=$ Carychium, Müller.

Jaminia a été également plusieurs fois préemployé avant Brown. Conovulus est de Lamarck et date de 1812 ; mais il ne s'applique pas aux espèces de ce groupe car il y comprend les $C$. bulimoides et $C$. coniformis qui en sont très distinctes.

Enfin dans son "Hist. Anim. s. Vert." (vi, pt. ii, 1822, pp. 136, 137). Lamarck écrit: "J'avais d'abord pensé que, parmi les coquilles à columelle plissée et dont l'ouverture n'est point échancrée à sa base, celles qui ont le bord droit, simple et tranchant étaient réellement fluviatiles et j'en avais fait un genre particulier sous le nom de Conovule (Conovulus). Mais ayant appris, d'après des observations qui m'ont été communiquées par M. Valenciennes, que mes conovules étaient des coquilles terrestres ; je supprime maintenant ce genre, et en réunis les espèces à celles de mes anciennes Auricules."

Il faut donc se résigner, en définitive, à adopter le nom récent de Myosotella pour ce groupe. 


\title{
$2 \mathrm{BHL}$ Biodiversity Heritage Library
}

\author{
Pallary, Paul. 1921. "QUELQUE RECTIFICATIONS DE NOMENCLATURE \\ CONCERNANT DES MOLLUSQUES DE LA FAUNE PALEARCTIQUE." Proceedings \\ of the Malacological Society of London 14, 141-147. \\ https://doi.org/10.1093/oxfordjournals.mollus.a063739.
}

View This Item Online: https://www.biodiversitylibrary.org/item/95719

DOI: https://doi.org/10.1093/oxfordjournals.mollus.a063739

Permalink: https://www.biodiversitylibrary.org/partpdf/202453

\section{Holding Institution}

Smithsonian Libraries

\section{Sponsored by}

Smithsonian

\section{Copyright \& Reuse}

Copyright Status: Public domain. The BHL considers that this work is no longer under copyright protection.

This document was created from content at the Biodiversity Heritage Library, the world's largest open access digital library for biodiversity literature and archives. Visit BHL at https://www.biodiversitylibrary.org. 